\title{
Simple Model of the Outflow from Starburst Galaxies: Application to Radio Observations
}

\author{
V.N.Zirakashvili \\ Institute for Terrestrial Magnetism, Ionosphere and Radiowave Propagation, 142190, Troitsk, Moscow \\ Region, Russia \\ Max-Planck-Institut für Kernphysik, Postfach 103980, 69029 Heidelberg, Germany \\ zirak@mpimail.mpi-hd.mpg.de \\ and \\ H.J.Völk \\ Max-Planck-Institut für Kernphysik, Postfach 103980, 69029 Heidelberg, Germany
}

\begin{abstract}
We present an analytical model for the hydrodynamic outflow from the disk of a starburst galaxy. The model is used to calculate the cosmic ray propagation and the radio intensity distribution in the nuclear starbust region of NGC 253. We find that the cosmic ray energy production rate of the central $600 \mathrm{pc}$ of NGC 253 is about $3 \cdot 10^{41} \mathrm{erg} \mathrm{s}^{-1}$, that is about 15 percent of the total mechanical supernova power. For this inner region we estimate a terminal outflow velocity of $900 \mathrm{~km} \mathrm{~s}^{-1}$ and a mass loss rate of $\dot{M}=2 \div 4 M_{\odot}$ per year.
\end{abstract}

Subject headings: cosmic rays- acceleration-galaxies

\section{Introduction}

Observations of the soft X-ray and radio continua and the $H_{\alpha}$ line emission support the idea of galactic winds from starburst galaxies (see e.g. Strickland (2004) for a review). In these galaxies which exibit a very high and spatially localised stellar formation rate, powerful stellar winds and supernova explosions produce a large amount of very hot gas. This gas expands out of the starburst region and forms a supersonic galactic wind flow.

Also in more quiescent galaxies such as the Milky Way galactic wind flows can arise. They are primarily driven by cosmic rays (Ipavich (1975); Breitschwerdt et al. (1987), (1991), (1993); Zirakashvili et al. (1996)). In such galaxies the gas heated by a spatially extended supernova activity loses its energy largely by radiative loses, whereas the nonthermal cosmic ray component, produced in the same context, can only cool adiabatically.
In addition, the cosmic rays produced in the galactic disk can not freely escape from the Galaxy but rather amplify Alfvén waves (e.g. Wentzel (1974)) that lead to an efficient dynamical coupling of the thermal gas to the energetic particle component (Ptuskin et al. (1997)). Therefore the cosmic ray pressure gradient is the dominant driver of such an extended galactic wind flow. Due to the different mechanisms these two types of outflow have somewhat different characteristics.

Chevalier \& Clegg ((1985)) introduced an elegant analytical hydrodynamic model for the galactic wind flow from a starburst galaxy. It assumes steady-state spherical symmetry. Despite its simplicity it seems qualitatively valid and is often used for estimates of the X-ray distribution and of the cosmic ray propagation.

However, the assumed spherical shape of the starburst region is a disadvantage of this model. In many cases the starburst region has an ellipsoidal shape or a disk geometry. They are more 
adequate for the problem considered. An analytical approach to this case is not simple because one should solve at least a two-dimensional system of hydrodynamic equations. For this reason a number of numerical calculations for galactic wind flows have been made (e.g. Habe \& Ikeuchi (1980); Tomisaka \& Ikeuchi (1988); Suchkov et al. (1994); Strickland \& Stevens (2000)). They take also the temporal evolution of the flow into account.

These studies evidently show that the powerful energy release in the starburst region produces forward and backward shocks propagating in the galactic halo. They move mainly perpendicular to the galactic disk. After 3-10 million years (depending on the surrounding halo gas density) the shocks break through into the intergalactic medium. At smaller distances the galactic wind flow becomes steady state. It is bounded by almost vertical walls of the shocked wind and halo gas (e.g. Suchkov et al. (1994)).

The hydrodynamic equations can be significantly simplified for so-called self-similar stationary flows. This means that different streamlines have the same shape but different scales. The equations then reduce to ordinary differential equations. This approach was first introduced by Bardeen \& Berger ((1978)) for galactic winds. It has been successfully used in the theory of collimated rotating magnetohydrodynamic flows from accretion disks and of active galactic nuclei (e.g. Blandford \& Payne (1984); Contopoulos \& Lovelace (1994)). In spite of rather stringent boundary conditions at the base of the flow in the form of a spatial power-law gas pressure dependence, it appears to us as a useful tool. Indeed many nuclear starbursts do show power-law pressure profiles at distances of 100 to $1000 \mathrm{pc}$ from the nucleus in the directions perpendicular and parallel to the major axis (e.g. Heckman et al. (1990)).

In this paper we shall use such a self-similar approach for the description of the single-fluid hydrodynamic outflow from a starburst galaxy. We have in particular found an explicit nontrivial analytic solution for one special (but not unreasonable) power-law dependence of the gas pressure at the base of the wind.

We apply the solution found to a calculation of the radio emission from the central $600 \mathrm{pc}$ of the starburst galaxy NGC253. The required additional transport equation for nonthermal rela- tivistic electrons couples the synchrotron emission morphology, intensity, and spectrum with the overall outflow dynamics. This permits a determination of the flow speed $u$ and of the cosmic ray energy production rate $\dot{E}_{\mathrm{CR}}$ as a measure of the total mechanical energy production $\dot{E}_{\mathrm{SN}} \sim 10 \dot{E}_{\mathrm{CR}}$ in supernovae, through a comparison with the radio synchrotron observations. We limit ourselves to this central region because a purely advective propagation model for the cosmic ray electrons is used. Such a model would not be adequate for the outer part of NGC253 since this outer region exhibits a steep radio spectrum (Hummel et al. (1984), Carilli et al. (1992)) where diffusion dominates advection. Our approach may be considered as complementary to other methods related with $\mathrm{X}$-ray and optical spectroscopic observations.

The basic equations are given in the next section. A one-dimensional model for a thin starburst region similar to Chevalier \& Clegg's model is described in section 3. Section 4 contains the analytic solution which we found. Applications of this solution to cosmic ray propagation in the galaxy NGC 253 are given in sections 5 and 6 . The outflow parameters derived are described in section 7. Section 8 contains a discussion of the results obtained together with our conclusions.

\section{Basic equations}

We shall assume that the starburst region lies in the galactic disk. The problem considered is mirror symmetric relative to the galactic midplane. The galactic wind flow originates in the disk and extends into the galactic halo. In this region the steady-state axially symmetric gasdynamic equations can be written in spherical coordinates $R$ and $\theta$ (the polar axis coinciding with the axis of galactic rotation):

$$
\begin{array}{r}
\left(u_{R} \frac{\partial}{\partial R}+\frac{u_{\theta}}{R} \frac{\partial}{\partial \theta}\right) u_{R}=-\frac{1}{\rho} \frac{\partial p}{\partial R}+\frac{u_{\theta}^{2}}{R} \\
\left(u_{R} \frac{\partial}{\partial R}+\frac{u_{\theta}}{R} \frac{\partial}{\partial \theta}\right) u_{\theta}=-\frac{1}{\rho R} \frac{\partial p}{\partial \theta}-\frac{u_{R} u_{\theta}}{R} \\
\left(u_{R} \frac{\partial}{\partial R}+\frac{u_{\theta}}{R} \frac{\partial}{\partial \theta}\right) \rho= \\
-\rho\left(\frac{1}{R^{2}} \frac{\partial}{\partial R}\left(R^{2} u_{R}\right)+\frac{1}{R \sin \theta} \frac{\partial}{\partial \theta}\left(\sin \theta u_{\theta}\right)\right)
\end{array}
$$




$$
\begin{gathered}
\left(u_{R} \frac{\partial}{\partial R}+\frac{u_{\theta}}{R} \frac{\partial}{\partial \theta}\right) p= \\
-\gamma p\left(\frac{1}{R^{2}} \frac{\partial}{\partial R}\left(R^{2} u_{R}\right)+\frac{1}{R \sin \theta} \frac{\partial}{\partial \theta}\left(\sin \theta u_{\theta}\right)\right)
\end{gathered}
$$

Here $u_{R}$ and $u_{\theta}$ are the radial and latitudinal components of the gas velocity, respectively, $\theta$ is the colatitude, $\rho$ and $p$ are the gas mass density and pressure, respectively, and $\gamma$ is the adiabatic index of the gas. The first two equations are the Euler equations of motion in the radial and latitude direction respectively. Eq.(3) is the continuity equation and Eq. (4) describes the evolution of the gas pressure.

We neglect gravity, galactic rotation and dynamical effects of the average magnetic field since they are insignificant for starburst outflows (cf. Chevalier \& Clegg (1985)). Magnetic fields and rotation were taken into account in the framework of self-similar solutions for winds from accretion disks and active galactic nuclei. Gravitational potentials in a simplified form can also be included (cf. Bardeen \& Berger (1978)).

We shall seek the solutions of Eqs. (1)-(4) in the following form:

$$
\begin{gathered}
u_{R}(R, \theta)=u_{R}(\theta) U(R / g(\theta)) \\
u_{\theta}(R, \theta)=u_{\theta}(\theta) U(R / g(\theta)) \\
\rho(R, \theta)=n(\theta) \rho_{0}(R / g(\theta)) \\
p(R, \theta)=n^{\gamma}(\theta) P(R / g(\theta))
\end{gathered}
$$

Here the functions $U\left(R_{0}\right), \rho_{0}\left(R_{0}\right)$, and $P\left(R_{0}\right)$ describe the spatial dependence of gas velocity, mass density and pressure at the base of the wind at $\theta=\pi / 2$, respectively, the function $g(\theta)$ determines the shape of streamlines, whereas the functions $u_{R}(\theta), u_{\theta}(\theta)$ and $n(\theta)$ describe the dependence of the radial, latitudinal velocity components and gas density along a streamline, respectively. We put $g(\pi / 2)=1$ and $n(\pi / 2)=1$. The equation for the streamlines is given by $R=R_{0} g(\theta)$. The equation for the function $g(\theta)$ may be written as

$$
\frac{\partial g}{\partial \theta} g^{-1}=u_{R} / u_{\theta}
$$

Substitution of Eqs. (5)-(8) into Eqs. (1)-(4) and taking into account Eq. (9) we obtain

$$
u_{\theta} \frac{\partial u_{R}}{\partial \theta}=-\frac{n^{\gamma-1}}{\rho_{0} U^{2}} P^{\prime} R_{0}+u_{\theta}^{2}
$$

$u_{\theta} \frac{\partial u_{\theta}}{\partial \theta}=-\frac{\gamma P}{\rho_{0} U^{2}} n^{\gamma-2} \frac{\partial n}{\partial \theta}+\frac{n^{\gamma-1}}{\rho_{0} U^{2}} P^{\prime} R_{0} \frac{\partial g}{\partial \theta} g^{-1}-u_{R} u_{\theta}$

$$
u_{\theta} \frac{\partial n}{\partial \theta}=-n\left(2 u_{R}+\frac{u_{\theta}}{\tan \theta}+\frac{\partial u_{\theta}}{\partial \theta}\right)
$$

Here ' denotes the derivative of the function $P$ with respect to its argument $R_{0}$. Eq.(4) is reduced to an identity. From Eqs. (9) and (12) we find that

$$
n g^{2} u_{\theta} \sin \theta=\text { const. }
$$

In order to separate the variables $R_{0}$ and $\theta$ we have to assume that at the wind base $P \propto \rho_{0} U^{2} \propto R_{0}^{\delta}$, with a constant $\delta<0$. This means that the gas pressure at the base is a power-law function of radius and that the ratio of dynamical pressure to the thermal pressure (the square of the flow Mach number) at the base is constant. Without loss of generality we can put $\rho_{0} U^{2} / P=1$. Note that $\rho_{0}\left(R_{0}\right)$ and $U\left(R_{0}\right)$ need not individually follow a power-low in $R_{0}$, but rather only the product $\rho_{0} U^{2}$. Now Eqs. (10) and (11) are reduced to

$$
\begin{gathered}
u_{\theta} \frac{\partial u_{R}}{\partial \theta}=-\delta n^{\gamma-1}+u_{\theta}^{2} \\
\left(u_{\theta}^{2}-\gamma n^{\gamma-1}\right) u_{\theta}^{-1} \frac{\partial u_{\theta}}{\partial \theta} \\
=n^{\gamma-1}\left(\frac{\gamma}{\tan \theta}+\frac{u_{R}}{u_{\theta}}(\delta+2 \gamma)\right)-u_{R} u_{\theta}
\end{gathered}
$$

Using Eqs. (9) and (13)-(15) one integral of the system can be found as

$$
\frac{u_{R}^{2}}{2}+\frac{u_{\theta}^{2}}{2}+\frac{\gamma}{\gamma-1} n^{\gamma-1}=\text { const }
$$

The Bernoulli constant for the each streamline differs from this integral by the factor $U^{2}(R / g(\theta))$.

As a result the problem is reduced to the solution of two ordinary differential equations (14) and (15) with the additional integral (16). If the solution is known, the function $g$ can be found from Eq. (13). Boundary conditions should be determined at the wind base at $\theta=\pi / 2$. Generally speaking, for arbitrary boundary values $u_{R}$ and $u_{\theta}$, the solutions considered do not occupy 
the whole space between $\theta=0$ and $\theta=\pi / 2$ (cf. Bardeen \& Berger (1978)). The boundary values should be adjusted such that the solution occupies the whole space.

As a natural boundary value we shall use $u_{R}=$ 0 at $\theta=\pi / 2$ for a starburst wind. It corresponds to a thin starburst region in the galactic disk. One can expect that the hot gas expands perpendicular to the disk in that case.

\section{Solution inside a thin starburst region}

If the starburst region has the form of thin disk, the one-dimensional hydrodynamic equations are a good approximation:

$$
\begin{gathered}
\frac{\partial}{\partial z} \rho u=q(z) \\
\rho u \frac{\partial u}{\partial z}=-\frac{\partial p}{\partial z}-q(z) u \\
\frac{\partial}{\partial z} \rho u\left(\frac{u^{2}}{2}+\frac{\gamma}{\gamma-1} \frac{p}{\rho}\right)=Q(z)
\end{gathered}
$$

Here $z$ is the height above disk midplane, $q(z)$ and $Q(z)$ are the sources of mass and energy, respectively. These equations are similar to the ones used by Chevalier \& Clegg ((1985)) but for a plane parallel case. We shall assume that the problem is symmetric relative to the galactic midplane.

Eqs. (17)-(19) can be integrated:

$$
\begin{gathered}
\rho u=\int_{0}^{z} d z^{\prime} q\left(z^{\prime}\right) \\
p=p(0)-u \int_{0}^{z} d z^{\prime} q\left(z^{\prime}\right) \\
\frac{u^{2}}{2}+\frac{\gamma}{\gamma-1} \frac{p}{\rho}=\frac{\int_{0}^{z} d z^{\prime} Q\left(z^{\prime}\right)}{\int_{0}^{z} d z^{\prime} q\left(z^{\prime}\right)}
\end{gathered}
$$

Here $p(0)$ is the gas pressure in the midplane. The substitution of $\rho$ and $p$ from the first two equations into the third gives a quadratic equation for the gas velocity $u$. Its solution is given by

$$
\begin{gathered}
u(z)=2 \frac{\gamma-1}{\gamma p(0)} \int_{0}^{z} d z^{\prime} Q\left(z^{\prime}\right) \\
\times\left(1+\sqrt{1-2 \frac{\gamma^{2}-1}{\gamma^{2} p^{2}(0)} \int_{0}^{z} d z^{\prime} Q\left(z^{\prime}\right) \int_{0}^{z} d z^{\prime} q\left(z^{\prime}\right)}\right)^{-1}
\end{gathered}
$$

The total energy and mass production rate per unit area of starburst disk are given by

$$
\begin{aligned}
Q_{0} & =2 \int_{0}^{h} d z Q(z), \\
q_{0} & =2 \int_{0}^{h} d z q(z) .
\end{aligned}
$$

Here $h$ is the halfwidth of the starburst disk. We can calculate the midplane gas pressure $p(0)$ if the Mach number $M=\sqrt{\rho u^{2} / \gamma p}$ at the boundary of the starburst disk $z=h$ is given. Its value is determined by the wind solution in the halo region (cf. next Section). E.g. $M=1$ in the Chevalier \& Clegg model ((1985)). Using Eqs. (20)-(22) we found the gas pressure, the gas mass density and the gas velocity at the boundary of the starburst disk

$$
\begin{gathered}
p(h)=\frac{p(0)}{1+\gamma M^{2}}=\frac{1}{2 \gamma M} \sqrt{\frac{2(\gamma-1) Q_{0} q_{0}}{2+(\gamma-1) M^{2}}} \\
\rho(h)=\frac{1}{2 M} \sqrt{\frac{q_{0}^{3}}{Q_{0}} \frac{2+(\gamma-1) M^{2}}{2(\gamma-1)}} \\
u(h)=M \sqrt{\frac{Q_{0}}{q_{0}} \frac{2(\gamma-1)}{2+(\gamma-1) M^{2}}}
\end{gathered}
$$

These quantities formally coincide with the corresponding quantities at the wind base in the thin disk approximation: $p(h)=p(\pi / 2), \rho(h)=$ $\rho(\pi / 2)$ and $u(h)=-u_{\theta}(\pi / 2)$. We should underline that this solution is rather general and is not related to self-similarity of the halo outflow. It is valid for any distribution of energy and mass sources $Q_{0}\left(R_{0}\right)$ and $q_{0}\left(R_{0}\right)$ in the galactic disk. However, the value of $M$ in general depends on $R_{0}$ and is determined by a solution in the halo region. For a starburst outflow $M$ is close to unity if the adiabatic index is not close to unity (see also the next Section).

\section{Analytical solution in the galactic halo}

In the case $\delta=-2 \gamma /(2 \gamma-1)$ an analytical solution of Eqs. (14)-(16) can be found in the first quadrant $0<\theta<\pi / 2$ :

$$
u_{R}=u_{\infty} \cos \theta
$$




$$
\begin{gathered}
u_{\theta}=-\left(1-\gamma^{-1}\right) u_{\infty} \sin \theta \\
n=\sin ^{2 /(\gamma-1)} \theta \\
g=\sin ^{-\gamma /(\gamma-1)} \theta
\end{gathered}
$$

Here the value of $u_{\infty}$ fixes the asymptotic velocity at distances $R$ that are large compared to the radius $R_{0}$ for each streamline $u_{\infty} U(R / g(\theta))$. The flow lines for this solution for $\gamma=5 / 3$ and $\delta=-10 / 7$ and the surfaces of constant Mach number are shown in Fig.1 in cylindrical coordinates $r$ and $z$. The initial Mach number of the flow is $M=\sqrt{2(\gamma-1) /(2 \gamma-1)}$. For other values of $\delta$ the solutions of Eqs. (14)-(16) can be obtained numerically. The streamlines for these solutions look similar to lines shown in Fig.1. For $\delta$ closer to zero the streamlines are closer to the axis, i.e. the wind is collimated more strongly. We have not found the solutions for $\delta<-2 \gamma /(2 \gamma-1)$ and for a natural boundary condition $u_{R}=0$ at $\theta=\pi / 2$. Therefore the analytical solution with $\delta=-2 \gamma /(2 \gamma-1)$ has the maximally opened flowlines in the framework of self-similar flows.

As was first noted by Bardeen \& Berger ((1978)) the equations for self-similar flow do not contain any particular points corresponding to sonic points of the flow. The only particular point is the point where the sonic velocity equals the latitudinal component of the flow velocity (cf. Eq. (15)). This component is always smaller than the sonic velocity in the solutions found. The wind starts at the base with subsonic velocity (initial Mach number $M \approx 0.756$ for $\gamma=5 / 3$ ). It is accelerated and passes through the sonic point at about $\theta \approx 70^{\circ}$ (see Fig.1). This picture corresponds to a flow in a Laval nozzle. The cross-section of the Laval nozzle shrinks in the subsonic part of the flow in order to reach sonic velocities and expands after the sonic point in order to obtain an acceleration of the flow beyond. As can be seen in Fig. 1 this is indeed the case. It is interesting that this flow geometry is organized by the gas itself through the perpendicular force balance. At the base of the wind the gas pushes the flowlines out of the axis. At larger heights the situation is reversed. The wind originating closer to the axis expands faster. The gas pressure drops adiabatically and the gas pressure of the wind originating at larger distances pushes the flow lines to the axis.

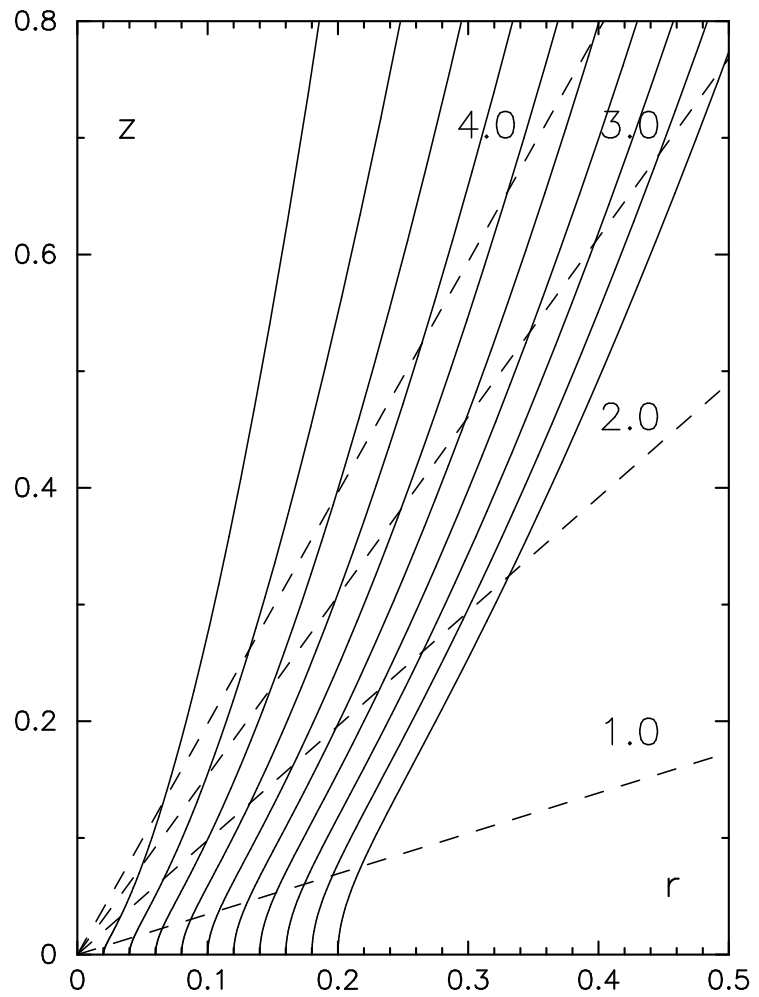

Fig. 1.- Flow lines of the analytical solution (solid lines) for $\gamma=5 / 3$ and $\delta=-10 / 7$ and surfaces of constant Mach number (dashed lines). The units of the cylindrical coordinates $r$ and $z$ are arbitrary. The Mach number values are shown near the corresponding dashed lines.

\section{Propagation of cosmic ray electrons in the wind}

We shall neglect the diffusion of energetic electrons in the starburst outflow. This seems reasonable since the flow velocity is quite high. In addition, the cosmic ray scattering produced by self-excited Alfvén waves (Ptuskin et al. (1997)) should be very effective in starbursts. This effect reduces the cosmic ray diffusion coefficient. The estimates of this effect in our Galaxy give for the diffusion coefficient the value $10^{28} \mathrm{~cm}^{2} \mathrm{~s}^{-1}$ for an energy of $10 \mathrm{GeV}$ (cf. Ptuskin et al. (1997)). It is inversely proportional to the cosmic ray production power per unit area of the galactic disk and is proportional to the magnetic field strength. Since starbursts have a high cosmic ray production power per unit area, typically a factor of 100 
to 1000 or more larger than in our Galaxy, and since the magnetic field is a factor of 10 larger, the diffusion coefficient in starbursts is smaller by at least a factor of 10 to 100, and the diffusion is negligible in comparison with advection for 10 $\mathrm{GeV}$ electrons which produce radio synchrotron emission ( for an application of these ideas to $\mathrm{TeV}$ gamma-ray emitting particles, see Aharonian et al. (2005)). The cosmic ray diffusion coefficient of much higher energy particles can in addition become quite small, if one takes into account that the starburst region is very turbulent, leading to additional strong magnetic field fluctuations which are produced by the turbulent velocity field.

Assuming azimuthal symmetry and a steady state the isotropic part of the cosmic ray energy distribution function $N(R, \theta, E)$ obeys the following equation

$$
\begin{gathered}
\left(u_{R} \frac{\partial}{\partial R}+\frac{u_{\theta}}{R} \frac{\partial}{\partial \theta}\right) N=\frac{\partial}{\partial E}(b(E) N) \\
+\frac{E^{3}}{3} \frac{\partial}{\partial E} \frac{N}{E^{2}}\left(\frac{1}{R^{2}} \frac{\partial}{\partial R}\left(R^{2} u_{R}\right)+\frac{1}{R \sin \theta} \frac{\partial}{\partial \theta}\left(\sin \theta u_{\theta}\right)\right)
\end{gathered}
$$

Here the second term on the right hand side describes the adiabatic energy losses, while $b(E)$ describes all other energy losses of particles. The boundary condition at the wind base $\theta=\pi / 2$ can be written as

$$
u_{\theta} \frac{E^{3}}{3} \frac{\partial}{\partial E} \frac{N_{0}}{E^{2}}-\frac{\partial}{\partial E}\left(b_{d}(E) N_{0}(E)\right)=\frac{1}{2} q_{C R}(E)
$$

Here $N_{0}(E)$ is the electron energy distribution at the wind base, $b_{d}(E)$ describes non-adiabatic energy losses in the thin starburst region and $q_{C R}(E)$ is the differential source energy spectrum of cosmic ray electrons normalized per unit area of the galactic disk.

The synchrotron emissivity $\epsilon_{\nu}$ can be written as

$$
\epsilon_{\nu}=\frac{\sqrt{3} B_{\perp} e^{3}}{4 \pi m c^{2}} \int d E N(E) f\left(\nu / \nu_{c}\right)
$$

Here $e$ and $m$ are the charge and the mass of an electron respectively, $B_{\perp}$ is the strength of magnetic field component perpendicular to the line of sight, $\nu$ is the radio-frequency, $c$ is the light velocity and $f$ is the function that describes the electron synchrotron emission (cf. Landau \& Lifshitz (1975)). The characteristic frequency of synchrotron radiation $\nu_{c}$ is given by the formula

$$
\nu_{c}=\frac{3 e B_{\perp}}{4 \pi m c}\left(\frac{E}{m c^{2}}\right)^{2}
$$

The synchrotron and inverse Compton energy losses can be written as

$$
b(E)=\frac{4}{3} c \sigma_{T}\left(U_{r a d}+\frac{B^{2}}{8 \pi}\right)\left(\frac{E}{m c^{2}}\right)^{2},
$$

where $\sigma_{T}=8 \pi e^{4} /\left(3 m^{2} c^{4}\right)$ is the Thompson crosssection, $B$ is the magnetic field strength and $U_{\text {rad }}$ is the radiation energy density.

We shall neglect bremsstrahlung and ionisation losses of electrons in the galactic wind flow. This is well justified, since the wind density is small. However, these losses may be non-negligible in the galactic disk which contains a large mass of gas. They are described by the second term on the left hand side of the boundary condition (34). The estimates show that these losses can be significant in the very center of a starburst. This depends on the gas mass that is rather uncertain. In addition it is not clear, whether radio electrons can well penetrate into gas clouds or whether they are quickly transported around clouds by the hot rarefied wind. The cosmic ray penetration into the gas clouds in the starburst region is beyond the scope of this paper. For the sake of simplicity we shall neglect these energy losses in the disk also.

The function $f$ can be expressed in terms of the Mc-Donald function. A so-called $\delta$-function approximation is often used for the function $f$. We shall use a more accurate method and approximate $f$ as (Pacholczyk (1970)):

$$
f(x)=1.81 x^{1 / 3} \mathrm{e}^{-x},
$$

where $x=\nu / \nu_{c}$.

The synchrotron intensity observed in a particular direction is given by the integral of the emissivity along the line of sight:

$$
J_{\nu}=\int d l \epsilon_{\nu}
$$


The spectrum of sources of cosmic ray electrons is given by

$$
q_{C R}(E)=\frac{K^{-1} Q_{C R}\left(R_{0}\right)}{E^{2} \ln \left(E_{\max } / E_{\min }\right)}
$$

where $Q_{C R}$ is the total power that is transfered by galactic sources to high energy cosmic ray protons with energies between $E_{\min }$ and $E_{\max }$ per unit area of the galactic disk, and $K$ is the proton to electron ratio in the source. It is expected that such a spectrum is formed in supernova remnants by the diffusive shock acceleration mechanism (Krymsky (1977), Axford et al. (1977), Bell (1978), Blandford \& Ostriker (1978)).

We shall neglect the production of secondary electrons and positrons by the nucleonic cosmic ray component in the dense gas disk. Although this effect can be essential for starbursts (cf. Paglione et al. (1996)), its magnitude is rather uncertain because of the uncertain gas mass and the uncertain degree of penetration of the $10 \div 100$ $\mathrm{GeV}$ cosmic nucleons into the gas clouds. If significant, this effect can be formally included in Eq. (40) by changing the proton to electron ratio $K$.

The solution of equation (33) with velocity $(5),(6)$ and energy losses $b(R, \theta, E)=b_{0}(R, \theta) E^{2}$ can be written as

$$
N(R, \theta, E)=N_{0}\left(E_{0}\right) \frac{E_{0}^{2}}{E^{2}} n^{4 / 3}(\theta)
$$

Here $E_{0}=E_{m} E /\left(E_{m}-E\right)$ and the maximum energy $E_{m}$ of electrons is given by

$$
\begin{aligned}
E_{m}^{-1}(R, \theta)= & -R \int_{\theta}^{\pi / 2} d \theta^{\prime} \frac{b_{0}\left(R g\left(\theta^{\prime}\right) / g(\theta), \theta^{\prime}\right)}{U(R / g(\theta)) u_{\theta}\left(\theta^{\prime}\right)} \\
& \times\left(\frac{n\left(\theta^{\prime}\right)}{n(\theta)}\right)^{1 / 3} \frac{g\left(\theta^{\prime}\right)}{g(\theta)}
\end{aligned}
$$

Using the analytical solution (30) and (32) and the source spectrum (40) we obtain

$$
\begin{aligned}
N(R, \theta, E)= & \frac{3 K^{-1} Q_{C R}\left(R \sin ^{\gamma /(\gamma-1)} \theta\right)}{8 E^{2} \ln \left(E_{\max } / E_{\min }\right)} H\left(E_{m}-E\right) \\
& \times \frac{\sin ^{8 / 3(\gamma-1)} \theta \gamma /(\gamma-1)}{u_{\infty} U\left(R \sin ^{\gamma /(\gamma-1)} \theta\right)}
\end{aligned}
$$

where $H(E)$ is the step function. The maximum energy $E_{m}$ is reduced to

$$
\begin{gathered}
E_{m}^{-1}=\frac{R \gamma /(\gamma-1)}{u_{\infty} U\left(R \sin ^{\gamma /(\gamma-1)} \theta\right)} \\
\times \int_{\theta}^{\pi / 2} \frac{d \theta^{\prime}}{\sin \theta^{\prime}} b_{0}\left(R\left(\frac{\sin \theta}{\sin \theta^{\prime}}\right)^{\frac{\gamma}{\gamma-1}}, \theta^{\prime}\right)\left(\frac{\sin \theta}{\sin \theta^{\prime}}\right)^{\frac{3 \gamma-2}{3(\gamma-1)}}
\end{gathered}
$$

\section{Modeling of the radio spectra of NGC 253}

NGC 253 is a nearby edge-on (inclination angle $i=78^{\circ}$; Pence (1981)) spiral starburst galaxy at distance $d=2.5 \mathrm{Mpc}$ (Mauersberger et al. (1996)). It has extended radio (cf. Carilli et al. (1992)) and X-ray (cf. e.g. Strickland (2004)) halos that appear to be related with the powerful superwind flow originating in the galactic nucleus. Our model of the outflow can be used to model the spatial X-ray and radio distribution in the central region of this galaxy.

We have performed the modeling of the radio distribution in the central 600 pc of NGC 253 using the results obtained in the previous sections. The radio spectrum of this galaxy is flat at distances $R_{0}<1 \mathrm{kpc}$ from the nucleus (Carilli et al. (1992)) with a spectral index larger than -0.6. We expect that in this region cosmic rays propagate advectively. Concretely, we choose the function $U\left(R_{0}\right)=1$. This means that the initial velocity is constant along the base of the wind and that the velocity depends on the colatitude $\theta$ only. The analytical solution found in the previous sections can be written for this case as

$$
\rho(R, \theta)=\rho_{g} \sin ^{-2 \frac{\gamma-1}{2 \gamma-1}} \theta\left(\frac{R}{R_{g}}\right)^{-\frac{2 \gamma}{2 \gamma-1}}
$$

$p(R, \theta)=\frac{1}{2 \gamma^{3}}(2 \gamma-1)(\gamma-1) \rho_{g} u_{\infty}^{2} \sin ^{\frac{2 \gamma}{2 \gamma-1}} \theta\left(\frac{R}{R_{g}}\right)^{-\frac{2 \gamma}{2 \gamma-1}}$

Here the asymptotic velocity $u_{\infty}$ is the same for all streamlines, $R_{g}$ is the radius of the wind base and $\rho_{g}$ is the gas density at the wind base at $R=R_{g}$. Dependence of velocity components on colatitude is given by Eqs. (29) and (30).

We use a radiation field distribution at $R<R_{g}$ in the simplified form: 


$$
U_{\mathrm{rad}}=\frac{L_{\mathrm{IR}}+L_{\mathrm{B}}}{4 \pi R_{g}^{2} c}\left(\frac{R}{R_{g}}\right)^{-\frac{2 \gamma}{2 \gamma-1}}
$$

Here $L_{\mathrm{IR}}$ and $L_{\mathrm{B}}$ are the infrared and starlight luminosities of the galaxy inside the region with wind base radius. It is reasonable to assume that radiation field is distributed similar to distribution of energy production in the starburst disk.

For a calculation of the synchrotron emissivity we need to define the magnetic field distribution. Radio observations of NGC 253 (Beck et al. (1994)) and many other galaxies show that the radio emission is weakly polarized. This means that the random component of the magnetic field is larger then the regular component. In the case of a starburst this random component can be generated by turbulence in the starburst disk and transported by the wind into the halo. Another possibility is to treat this random magnetic field as large amplitude Alfvén waves propagating along the weak regular field and generated by the motions of the footpoints of the field lines in the starburst disk. This picture is similar to the case of the solar corona.

We shall treat the random magnetic field as a fluid with an adiabatic index $\gamma_{m}$. It is known that Alfvén waves have the adiabatic index $\gamma_{m}=3 / 2$ (Dewar (1970), McKee \& Zweibel (1995)); an isotropic random magnetic field has the adiabatic index $\gamma_{m}=4 / 3$ (Mestel (1965)). We used the following expression for the magnetic field which has a similar R-dependence as $p(R, \theta)$ (cf. Eq. (46)):

$$
B=B_{0}(\sin \theta)^{\frac{1}{\gamma-1}\left(\gamma_{m}-\frac{\gamma^{2}}{2 \gamma-1}\right)}\left(\frac{R}{R_{g}}\right)^{-\frac{\gamma}{2 \gamma-1}}
$$

Here $B_{0}$ is the magnetic field strength at $R=R_{g}$. The magnetic field component $B_{\perp}$ in Eqs. (35) and (36) was calculated as $B_{\perp}=\sqrt{2 / 3} B$.

The distribution of cosmic ray sources in the starburst disk in Eq. (43) was taken in a similar form

$$
Q_{\mathrm{CR}}\left(R_{0}\right)=\frac{\gamma-1}{2 \gamma-1} \frac{L_{\mathrm{CR}}}{\pi R_{g}^{2}}\left(\frac{R_{0}}{R_{g}}\right)^{-\frac{2 \gamma}{2 \gamma-1}},
$$

where $L_{\mathrm{CR}}$ is the cosmic ray energy production rate of the starburst.
The continuum radio emission of galaxies consists of two main components: synchrotron emission of cosmic ray electrons and a thermal emission of the ionized gas which is produced mainly in HII regions, ionized by OB stars (see e.g. Condon (1992) for a review). The thermal emission is closely related with thermal (so-called free-free) absorption which can be significant at low frequencies and can produce a flattening and even a turnover of the spectrum. It appears that this indeed the case for the spectrum of the nucleus of NGC 253 (Carilli (1996)). Other possible reasons for the flattening are reacceleration of low-energy electrons (Pohl \& Schlickeiser (1990)), and ionisation and bremsstrahlung energy losses of electrons in the starburst disk (Hummel, (1991)). We give preference to free-free absorption here, since the thermal component of the radio emission is undoubtedly present in NGC 253.

To take free-free absorption into account we shall assume that the thin galactic disk seen faceon has the following distribution of the absorption depth

$$
\tau\left(R_{0}\right)=\tau_{0}\left(\frac{R_{0}}{R_{g}}\right)^{-\frac{2 \gamma}{2 \gamma-1}}\left(\frac{1 \mathrm{GHz}}{\nu}\right)^{2.1} .
$$

Here $\tau_{0}$ is the absorption depth of the disk at 1 $\mathrm{GHz}$ and at $R_{0}=R_{g}$. It was assumed that this distribution is similar to the distribution of sources of energy in the galactic disk. This is reasonable, since the absorbing gas is ionized by OB stars. The number of these stars is proportional to the input of energy. The inclined disk of NGC 253 absorbs the synchrotron emission of the northern part of the halo.

The thermal emission brightness of the inclined disk can be expressed in terms of the absorption depth $\tau$ and the inclination angle $i$ :

$$
J_{\mathrm{th}}=2 \frac{T \nu^{2}}{c^{2}}\left(1-\mathrm{e}^{-\tau /|\cos i|}\right) .
$$

Here $T$ is the absorbing gas temperature in energetic units. Generally speaking, the two last equations are valid if the absorbing gas has a large covering factor. This is possibly not the case in the disk of NGC 253. However it is possible in the central region, where the main part of the absorption occurs.

We should take into account the synchrotron emission of supernova remnants (SNRs) in the 
starburst disk. The SNRs in the disk have a small volume filling factor. However, the magnetic field strength and the number density of relativistic electrons in SNRs are larger in comparison with the corresponding quantities in the interstellar medium of the starburst disk and in the wind halo. We used the following expression for the brightness of the radio emission produced by the SNRs from the inclined disk:

$$
\begin{aligned}
J_{\mathrm{SNR}}=\frac{\gamma-1}{2 \gamma-1} & \frac{J_{0} d^{2}}{\pi R_{g}^{2} \tau}\left(\frac{R_{0}}{R_{g}}\right)^{-\frac{2 \gamma}{2 \gamma-1}}\left(\frac{\nu}{1 \mathrm{GHz}}\right)^{-0.5} \\
& \times\left(1-\mathrm{e}^{-\tau /|\cos i|}\right)
\end{aligned}
$$

This emission from SNRs is also influenced by freefree absorption. The total radio flux $J_{0}$ from SNRs is about one tenth of the total radio flux for normal galaxies (Condon (1992)), but it can be larger in galaxies with a fast escape of electrons. A more quantitative estimate was obtained by Ulvestad ((1982)) using the relation between radio surface brightness and diameter (the ${ }^{\prime \prime} \Sigma-D^{\prime \prime}$ relation, see also Berezhko \& Völk (2004)):

$$
\begin{gathered}
J_{0}=206 \mathrm{Jy}\left(\frac{d}{\mathrm{Mpc}}\right)^{-2}\left(\frac{\nu_{\mathrm{SN}}}{1 \mathrm{yr}^{-1}}\right) \\
\times\left(\frac{E_{\mathrm{SN}}}{10^{50 \mathrm{erg}}}\right)^{-1 / 17}\left(\frac{n_{\mathrm{ISM}}}{1 \mathrm{~cm}^{-3}}\right)^{-2 / 17}
\end{gathered}
$$

where $\nu_{\mathrm{SN}}$ is the supernova rate. This flux weakly depends on the interstellar medium number density $n_{\mathrm{ISM}}$ and the supernova explosion energy $E_{\mathrm{SN}}$. We recalculated the numerical factor in Eq. (53) using the $\Sigma-D$ relation for supernovae in the starburst galaxy M82 (Huang et al. (1994)). It is a factor of two larger than the factor obtained by Ulvestad ((1982)). That means that supernovae in starburst galaxies are slightly brighter than in normal galaxies. This number is in a good agreement with radio observations of galaxies (cf. Condon (1992) for a review) and theoretical estimates (Lisenfeld \& Völk (2000)).

We have performed the modeling of the starburst wind with a radius $R_{g}=300 \mathrm{pc}$ and have calculated synchrotron and thermal emission using the formulae given in this and previous sections. The choice of this radius was done in accordance with the size of the region where the radio emission of NGC253 is concentrated (Ulvestad (2000)).
For the adiabatic index of the random magnetic field we use the adiabatic index of Alfvén waves $\gamma_{m}=3 / 2$. Since cosmic ray and Alfvén wave pressures are not negligible (see below) we also used a gas adiabatic index $\gamma=3 / 2$. The temperature of the absorbing gas was fixed at $8 \cdot 10^{3}$ K. The infrared luminosity of the nucleus of NGC 253 was taken as $L_{\mathrm{IR}}=1.24 \cdot 10^{10} L_{\odot}($ Radovich et al. (2001)). For the starlight luminosity, we used the luminosity of the bulge of NGC 253, $L_{\mathrm{B}}=0.39 \cdot 10^{10} L_{\odot}($ Pence $(1980))$. Also a source proton-to-electron ratio $K=70$ was chosen. Finally, a maximum energy $E_{\max }=10^{6} \mathrm{GeV}$ of the cosmic ray protons was assumed. The results are shown in Fig.2 and Fig.3.

The contour plot of the $1.4 \mathrm{GHz}$ brightness distribution modeled is shown in Fig.2. The radio distribution was convolved with a Gaussian beam with FWHM $5.1^{\prime \prime} \times 3.8^{\prime \prime}(62 \times 46 \mathrm{pc}$ at $2.5 \mathrm{Mpc}$ distance), and an inclination angle of $i=78^{\circ}$ was used. The distribution is slightly asymmetric relative to the horizontal axis due to free-free absorption. The comparison with the observed distribution (Mohan et al. (2005)) permits us to estimate the wind velocity. We found that the wind velocity $u_{\infty}$ should be larger than $500 \mathrm{~km} \mathrm{~s}^{-1}$. For smaller velocities the brightness drops too fast with height above midplane because of strong synchrotron and inverse Compton losses. We have found a satisfactory fit for $L_{C R}=2.94 \cdot 10^{41} \mathrm{erg} \mathrm{s}^{-1}, u_{\infty}=900$ $\mathrm{km} \mathrm{s}^{-1}, \tau_{0}=0.05|\cos i|, J_{0}=1.0 \mathrm{Jy}$ and $B_{0}=50$ $\mu \mathrm{G}$. The magnetic field pressure was taken close to the cosmic ray pressure. The value of the radio flux $J_{0}$ from SNRs corresponds to the supernova rate $\nu_{\mathrm{SN}}=0.06 \mathrm{yr}^{-1}$ which is intermediate between the value $\nu_{\mathrm{SN}}=0.03 \mathrm{yr}^{-1}$ of Matila \& Meikle $((2001))$ and $\nu_{\mathrm{SN}}=0.1 \mathrm{yr}^{-1}$ (Ulvestad \& Antonucci (1997)). Finally, a mean number density $n_{\mathrm{ISM}}=100 \mathrm{~cm}^{-3}$ of the interstellar medium in NGC253 (Paglione et al. (1995)), and a mean energy of supernova explosions $E_{\mathrm{SN}}=10^{51} \mathrm{erg}$ were assumed in Eq. (53).

The uncertainty of the velocity derived in this way is larger from the side of higher velocities. For example, for $u_{\infty}=500 \mathrm{~km} \mathrm{~s}^{-1}$ the contour lines near the upper and lower parts of the plot are shifted by about one contour level in the midplane direction. A similar shift in the opposite direction corresponds to $u_{\infty}=2000 \mathrm{~km} \mathrm{~s}^{-1}$. The derived value $u_{\infty}=900 \mathrm{~km} \mathrm{~s}^{-1}$ produces a good simi- 
larity between observed and modeled radio distribution in the southern part of the outflow. We think that the radio distribution in the northern part of the outflow should be explained by using a more complex model with slightly larger and variable wind velocities along the base (for example, another function $U\left(R_{0}\right)$ might be used). This can produce spur-like features in the observed radio distribution in the northern part of the outflow. Another possible explanation is limb-brightening due to the superwind's lateral interaction with halo gas which is not included in our model.

The spectrum of the NGC 253 nuclear region is shown in Fig.3 (solid line). The data points with the same resolution $33^{\prime \prime} \times 21^{\prime \prime}(400 \times 255 \mathrm{pc}$ at 2.5 Mpc distance) are taken from Carilli ((1996)).

The total radio flux of SNRs amounts to about one third of the total radio flux at $1.4 \mathrm{GHz}$. The thermal emission amounts to one third of the total intensity at $10 \mathrm{GHz}$. This is in good agreement with results for the separation of the thermal and nonthermal emission for the whole galaxy NGC 253 (Niklas et al. (1997)). The fit can be better if a smaller temperature of the emitting gas is used. It should be noted that Israel \& Mahoney ((1990)) found that the flattening of the radio spectra of galaxies at small frequencies can be explained by free-free absorption in a hypothetical cold ionized gas.

The observed synchrotron intensity mainly constrains the ratio of the cosmic ray power $L_{\mathrm{CR}}$ to the wind velocity. The wind velocity and cosmic ray power influence the spectral shape of the radio emission only weakly.

The cosmic ray luminosity found corresponds to the cosmic ray pressure at the wind base (at $\left.R=R_{g}\right)$ :

$$
p_{C R}=\frac{\gamma}{8(2 \gamma-1)} \frac{L_{C R}}{\pi u_{\infty} R_{g}^{2}}
$$

This gives $p_{\mathrm{CR}}=1.08 \cdot 10^{-10} \mathrm{erg} \mathrm{cm}^{-3}$ at the wind base at $R_{0}=R_{g}$. The total nonthermal (cosmic ray + Alfvén waves) luminosity is about $5.6 \cdot 10^{41}$ $\operatorname{erg~s}^{-1}$.

\section{Estimates of the outflow parameters}

We have modificated the Chevalier \& Clegg model ((1985)) for a thin starburst region (cf. Sect.3). In the particular case of the analytical

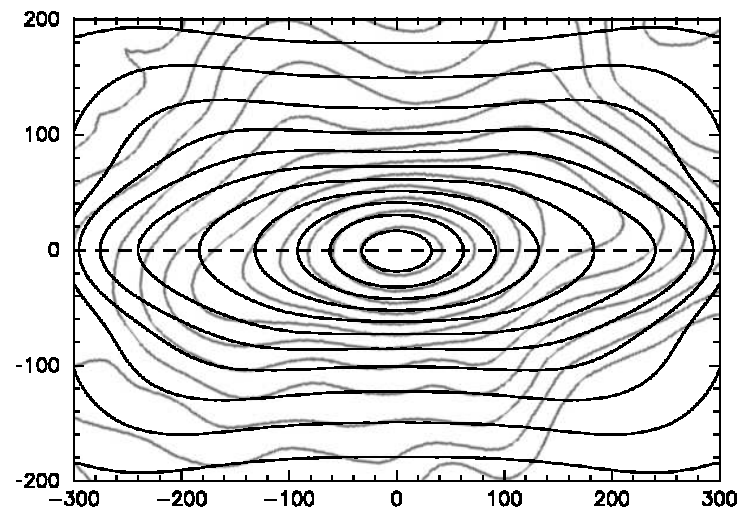

Fig. 2.- Radio brightness distribution of NGC 253 at $1.4 \mathrm{GHz}$ calculated in our model. The modeled radio intensity was convolved with a Gaussian beam of FWHM $5.1^{\prime \prime} \times 3.8^{\prime \prime}(62 \times 46 \mathrm{pc}$ at $2.5 \mathrm{Mpc}$ distance). The solid contour levels are a geometric progression in 1.7 , ending at $0.446 \mathrm{Jy} /$ beam in the center. The projected distance in $\mathrm{pc}$ is shown on both axes. The observed brightness distribution at $1.4 \mathrm{GHz}$ (Mohan et al. (2005)) is shown by the gray scale contours.

solution $(29),(30),(45),(46)$, the Eqs. (26) - (28) can be rewritten as

$$
\begin{gathered}
u_{\infty}=\frac{\gamma}{\gamma-1} u(h)=5.63 \cdot 10^{8} \mathrm{~cm} \mathrm{~s}^{-1} \\
\times\left(\frac{\dot{E}}{10^{43} \mathrm{erg} \mathrm{s}^{-1}}\right)^{1 / 2}\left(\frac{\dot{M}}{M_{\odot} \mathrm{yr}^{-1}}\right)^{-1 / 2}, \quad(55) \\
\rho(h)=1.88 \cdot 10^{-27} \mathrm{~g} \mathrm{~cm}^{-3} \frac{\gamma}{2 \gamma-1}\left(\frac{\dot{E}}{10^{43} \mathrm{erg} \mathrm{s}^{-1}}\right)^{-1 / 2} \\
\times\left(\frac{\dot{M}}{M_{\odot} \mathrm{yr}^{-1}}\right)^{3 / 2}\left(\frac{R_{g}}{1 \mathrm{kpc}}\right)^{-2}\left(\frac{R_{0}}{R_{g}}\right)^{-\frac{2 \gamma}{2 \gamma-1}}, \\
p(h)=2.98 \cdot 10^{-10} \mathrm{erg} \mathrm{cm}^{-3} \frac{\gamma-1}{\gamma^{2}}\left(\frac{\dot{E}}{10^{43} \mathrm{erg} \mathrm{s}^{-1}}\right)^{1 / 2} \\
\times\left(\frac{\dot{M}}{M_{\odot} \mathrm{yr}^{-1}}\right)^{1 / 2}\left(\frac{R_{g}}{1 \mathrm{kpc}}\right)^{-2}\left(\frac{R_{0}}{R_{g}}\right)^{-\frac{2 \gamma}{2 \gamma-1}},
\end{gathered}
$$

Here $\dot{E}$ and $\dot{M}$ are the total energy and mass production rates of the starburst disk at $R<R_{g}$. 


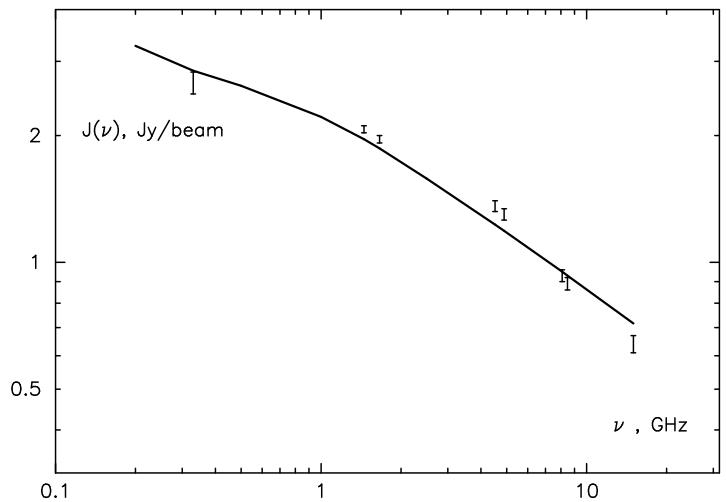

Fig. 3.- Spectrum of the nuclear region of NGC 253 (solid curve), calculated in our model. The modeled radio intensity was convolved with a Gaussian beam of FWHM $33^{\prime \prime} \times 21^{\prime \prime}(400 \times 255$ pc at $2.5 \mathrm{Mpc}$ distance). The data points are taken from Carilli ((1996)).

The energy production rate is determined mainly by supernovae. The upper limit for the supernova rate in the nucleus of NGC 253 is 0.3 $\mathrm{yr}^{-1}$ (Ulvestad \& Antonucci (1997)). It seems that the actual number is not larger than $0.1 \mathrm{yr}^{-1}$ because of the absence of a new supernova during the last years. For a supernova rate $0.06 \mathrm{yr}^{-1}$ in NGC 253 the energy production rate $\dot{E}=1.9 \cdot 10^{42}$ erg s ${ }^{-1}$ under the assumption of negligible radiative losses of the gas. The nonthermal luminosity found in the previous section is about one quarter of this number.

Since part of the supernova energy can be lost radiatively, the energy input may be smaller. The X-ray observations give an the energy input $\dot{E} \approx$ $10^{42} \mathrm{erg} \mathrm{s}^{-1}$ (Strickland et al. (2002)) and an average wind velocity $u$ between 400 and $500 \mathrm{~km}$ $\mathrm{s}^{-1}$ that is between the velocity $300 \mathrm{~km} \mathrm{~s}^{-1}$ at the wind base and the asymptotic velocity $900 \mathrm{~km} \mathrm{~s}^{-1}$ found in the previous section.

For this case we found the mass-loss rate $\dot{M}=$ $3.9 M_{\odot} \mathrm{yr}^{-1}$, and the total pressure $p=4.6 \cdot 10^{-10}$ erg $\mathrm{cm}^{-3}$ at $R=R_{g}$ and a factor of 15 larger at a distance of $50 \mathrm{pc}$ from the nucleus. These numbers should be compared with the cosmic ray and magnetic pressures derived in the previous section $p_{\mathrm{CR}}=1.1 \cdot 10^{-10} \mathrm{erg} \mathrm{cm}^{-3}$ and $B_{0}^{2} / 8 \pi=1.0 \cdot 10^{-10}$ erg $\mathrm{cm}^{-3}$ respectively. Therefore the nonthermal pressure is almost one half of the total pressure. The rest can be attributed to the thermal gas pres- sure, that is $p_{g}=2.5 \cdot 10^{-10} \mathrm{erg} \mathrm{cm}^{-3}$. The corresponding mass density is $\rho_{g}=3.8 \cdot 10^{-25} \mathrm{~g}$ $\mathrm{cm}^{-3}$. The corresponding temperature of a onephase fully ionized gas is about $5 \cdot 10^{6} \mathrm{~K}$.

This is not surprising. If 15 procent of the supernova energy is converted into cosmic ray energy and the rest is partially radiated, then the gas pressure may be comparable with the cosmic ray pressure. In this case it is reasonable to use a smaller adiabatic index $\gamma<5 / 3$ of the gas to include cosmic rays and magnetic fields.

In reality, a one-phase thermal gas with a temperature of several million degrees is excluded by the X-ray observations, since it would produce too large an amount of X-rays (Strickland et al. (2002)). The main fraction of the thermal energy may rather be contained in an even hotter gas component, and the main part of the mass may then be contained in gas with a temperature below one million degrees.

We can even consider an extreme case by assuming that the gas radiates all its energy. The superwind flow is then driven by cosmic rays and Alfvén waves (or turbulent magnetic fields) alone. Using a nonthermal luminosity $\dot{E}=5.6 \cdot 10^{41} \mathrm{erg}$ $\mathrm{s}^{-1}$, as found in the last section, we then obtain a mass-loss rate of $\dot{M}=2.2 M_{\odot} \mathrm{yr}^{-1}$.

\section{Conclusion}

We have presented a simple analytical model for a steady state axially symmetric gas-dynamic outflow from a starburst galaxy. In the case of a power-law radial distribution of the gas pressure at the wind base self-similar solutions can be found. For the special choice of the power-law index $\delta=$ $-2 \gamma /(2 \gamma-1) \approx 1.5$ we found an explicit analytical solution. This value of the index seems reasonable, since many starbursts show a strong concentration of the energy release towards the center.

The solution found has less steep pressure and density profiles in comparison with the Chevalier \& Clegg model (Chevalier \& Clegg (1985)). It is well known that the Chevalier \& Clegg solution is in agreement with the X-ray brightness of M82 at distances larger than 1-2 kpc from the nucleus, whereas at smaller distances the observed brightness relief is lower (e.g. Fabbiano (1988)). This may be understood in the framework of our model. At distances smaller than the starburst region gas 
collimation is possible. This results in weaker dependencies on radius. At larger distances the wind geometry is roughly spherically symmetric, perpendicular forces play no role and the Chevalier \& Clegg model is more adequate.

In reality the power-law pressure dependence can not be valid at small radii either, i.e. there exists some minimum radius. The wind originating inside this radius is not described by the selfsimilar solution. However, for a small minimum radius it blows in a narrow region near the axis. Therefore this restriction appears not to be essential.

The solution found was applied to the modeling of the radio distribution of the central part $(R<300 \mathrm{pc})$ of the starburst galaxy NGC 253 . This galaxy contains a very bright radio nucleus with a flat spectrum (with spectral index rnaging from -0.43 to -0.17 at different frequencies) and a less bright extended radio disk with a steeper spectrum of spectral index -0.7 (Hummel et al. (1984), Carilli et al. (1992)).

The propagation of the radio electrons can be purely advective in the central part of NGC 253 since the gas velocity is rather high (about 1000 $\mathrm{km} \mathrm{s}^{-1}$ ). It appears that this velocity is smaller in the outer part of the galactic disk $(R>1 \mathrm{kpc})$ where the spectrum is steeper. It is possible that the outer part contains a cosmic ray driven galactic wind which has small velocity at the wind base (cf. Breitschwerdt et. al. (1991); Zirakashvili et al. (1996)) and where the diffusion of particles results in the spectral steepening.

Our model is applicable for the central superwind region $(R<300 \mathrm{pc})$ of NGC253 which contains the central starburst. NGC253 has also a comparable energy release at larger distances. However this is distributed over an area which is at least a factor 100-1000 larger than the central starburst. Our model does not describe that region which contains a quasi-static galactic halo or a slow cosmic-ray driven galactic wind. It also does not describe the interaction of the gas in this region with the superwind flow.

We have obtained a satisfactory fit for the radio brightness distribution and for the radio spectrum of the nucleus of NGC 253. This permits us to estimate the parameters of the outflow: the wind velocity and mass loss rate of the starburst.
From a physics point of view these quantities should be determined from a theoretical model which describes how thermalised supernova ejecta and the interstellar gas heated by supernova shocks radiate some part of the energy and form an outflow from the starburst disk. In the absence of such a theory we limit ourself to the determination of these quantities from observations.

We found an asymptotic velocity of $900 \mathrm{~km} \mathrm{~s}^{-1}$ from this inner region. For an energy input of $10^{42} \mathrm{erg} \mathrm{s}^{-1}$ in the starburst nucleus of NGC253 we obtain a mass loss rate of $4 M_{\odot} \mathrm{yr}^{-1}$. This number drops down to $2 M_{\odot} \mathrm{yr}^{-1}$ for the extreme case when the gas radiates almost all its energy. Even this case does not contradict the X-ray observations, since it appears that the X-rays are produced by a hot gas that is formed from the interaction between the superwind and the circumstellar medium (halo gas, clouds etc.) (cf. Strickland et al. (2002)).

The cosmic ray production power is estimated as 15 procent of the supernova power. We found that cosmic ray and magnetic pressures are comparable with the gas pressure. Therefore we can conclude that the starburst outflow in NGC 253 is collectively driven by hot gas, cosmic rays and turbulent magnetic fields.

We thank the anonymous referee for a number of valuable suggestions regarding the interpretation of the theoretical results.

\section{REFERENCES}

Aharonian, F., Akhperjanian, A.G., Bazer-Bachi, A.R., et al. 2005, A\&A in press (arXiv:astro$\mathrm{ph} / 0507370$ )

Axford, W.I., Leer, E., Scadron, G., 1977, Proc. 15th Int. Cosmic Ray Conf., Plovdiv, 90, 937

Bardeen, J.M., \& Berger, B.K. 1978, ApJ 221, 105

Beck R., Carilli, C.L., Holdaway, M.A., \& Klein, U. 1994, A\&A 106,112

Bell, A.R., 1978, MNRAS, 182, 147

Berezhko, E.G., \& Völk, H.J. 2004, A\&A 427, 525

Blandford, R.D., \& Ostriker, J.P. 1978, ApJ, 221, L29 
Blandford, R.D., \& Payne, D.G. 1982, MNRAS 199,883

Breitschwerdt, D., McKenzie, J.F., \& Völk, H.J. 1987, Proc. 20th ICRC 2,115

Breitschwerdt, D., McKenzie, J.F., \& Völk, H.J. 1991, A\&A 245, 79

Breitschwerdt, D., McKenzie, J.F., \& Völk, H.J. 1993, A\&A 269, 54

Carilli, C.L., Holdaway, M.A., Ho, P.T.P., \& De Pree, C.G. 1992, ApJ 399, L59

Carilli, C.L. 1996, A\&A, 305, 402

Chevalier, R.A., \& Clegg, A.W. 1985, Nature 317, 44

Condon, J.J. 1992, ARA\&A, 30, 575

Contopoulos, G., \& Lovelace R.V.E. 1994, ApJ 429, 139

Dewar, R.L. 1970, Phys. Fluids, 13, 2710

Fabbiano, G. 1988, ApJ, 330, 672

Habe, A., \& Ikeuchi, S., 1980, Progr. of Theor. Phys. 64, 1995

Heckman, T.M., Armus, L., \& Miley, G.K. 1990, ApJS 74, 833

Huang, Z. P., Thuan, T. X., Chevalier, R. A., Condon, J. J., Yin, Q. F. 1994, ApJ, 424, 114

Hummel, E., Smith, P., \& van der Hulst, J.M. 1984, A\&A, 137, 138

Hummel, E. 1991, A\&A, 251, 442

Israel, F.P., \& Mahoney, M.J., 1990, ApJ, 352, 30

Ipavich, F. 1975, ApJ, 196, 107

Krymsky, G.F. 1977, Soviet Physics-Doklady, 22, 327

Landau, L.D., \& Lifshitz, E.M., The classical theory of fields 1975, Oxford: Pergamon Press

Lisenfeld, U., \& Völk, H.J. 2000, A\&A 354, 423

Matila, S., \& Meikle, W.P.S. 2001, MNRAS, 324, 325
Mauersberger, R., Wilson, T.L., Rood, R.T., Bania, T.M., Hein, H., \& Linhart, A. 1996, A\&A 306,141

McKee, C.F., Zweibel, E.G. 1995, ApJ, 440, 686

Mestel, L. 1965, Quart. J. Roy. Astr. Soc., 6, 161, 265

Mohan, N.R., Goss, W.M., \& Anantharamaiah, K.R. 2005, A\&A, 432, 1

Niklas, S., Klein, U., \& Wielebinski, R. 1997, A\&A, 322, 19

Pacholczyk, A.G. Radio astrophysics. Nonthermal processes in galactic and extragalactic sources, San Francisko: Freeman, 1970

Paglione, T.A.D., Tosaki, T., Jackson, J.M. 1995, ApJ, 454, L117

Paglione, T.A.D., Marscher, A.P., Jackson, J.M., \& Bertsch, D.L. 1996, ApJ, 460, 295

Pence, W.D. 1980, ApJ, 239, 54

Pence, W.D. 1981, ApJ, 247, 473

Pohl, M., \& Schlickeiser, R. 1992, A\&A, 263, 37

Ptuskin, V.S., Völk, H.J., Zirakashvili, V.N., \& Breitschwerdt, D. 1997, A\&A 321, 434

Radovich, M., Kahanpää, J., Lemke, D. 2001, A\&A, 377,73

Strickland, D.K. 2004, Proc. IAU Symp. 222, Th. Storchi Bergmann, L.C. Ho \& H.R. Schmitt, eds.

Strickland, D.K., Heckman, T.M., Weaver, K.A., Hoopes, C.G., \& Dahlem, M. 2002, ApJ 568, 689

Strickland, D.K., \& Stevens, I.R. 2000, MNRAS 314,511

Suchkov, A.A., Berman, V.G., Heckman, T.M., \& Balsara, D.S. 1994, ApJ 463, 528

Tomisaka, K., \& Ikeuchi, S. 1988, ApJ 330, 695

Ulvestad, J.S. 1982, ApJ, 259, 96

Ulvestad, J.S., \& Antonucci, R.R. 1997, ApJ, 488, 621 
Ulvestad, J.S. 2000, AJ, 120, 278

Wentzel, D.G. 1974, ARA\&A 12, 71-96

Zirakashvili, V.N., Breitschwerdt, D., Ptuskin, V.S., \& Völk, H.J. 1996, A\&A 311, 113-126

This 2-column preprint was prepared with the AAS IATEX macros v5.2. 